\title{
Aesthetic Outcome After Reconstruction of Complex Soft Tissue Defects with Free Antero-Lateral Thigh Flap Using Simple Equipment
}

\author{
Ashraf H. Abbas ${ }^{1}$, Moustafa Elmasry ${ }^{2}$, , Ingrid Steinvall ${ }^{3}$, Osama A. Adly ${ }^{1}$, \\ Mohamed A. Elbadawy ${ }^{1}$, Taha Ali Moati ${ }^{4}$, Folke Sjöberg ${ }^{5}$ \\ ${ }^{1}$ Plastic Surgery Unit, Surgery Dept., Suez Canal University, Ismailia, Egypt \\ ${ }^{2}$ Plastic Surgery Unit, Surgery Dept., Suez Canal University, Ismailia, Egypt and The Burn Centre Dept. of Hand and Plastic Surgery and, \\ Dept. of Clinical and Experimental Medicine, Linköping University, Linköping, Sweden \\ ${ }^{3}$ Burn Centre Department of Hand and Plastic Surgery, Linköping University, Linköping, Sweden \\ ${ }^{4}$ General Surgery department, Suez Canal University, Ismailia, Egypt \\ ${ }^{5}$ Burn Centre Depts. of Hand, Plastic Surgery and Intensive Care and Dept. of Clinical and Experimental Medicine, Linköping University, \\ Linköping, Sweden
}

\section{Email address:}

moustafa.elmasry@regionostergotland.se (M. Elmasry), mostafaelmasry@yahoo.com (M. Elmasry)

\section{To cite this article:}

Ashraf H. Abbas, Moustafa Elmasry, Ingrid Steinvall, Osama A. Adly, Mohamed A. Elbadawy, Taha Ali Moati, Folke Sjöberg. Aesthetic Outcome After Reconstruction of Complex Soft Tissue Defects with Free Antero-Lateral Thigh Flap Using Simple Equipment. Journal of Surgery. Special Issue: Postoperative Pain Syndrome. Vol. 3, No. 2-1, 2015, pp. 36-41. doi: 10.11648/j.js.s.2015030201.18

\begin{abstract}
Aim: We aimed to assess the aesthetic outcome of surgical reconstruction by free ALT flap using binocular singlerefraction magnifying glasses and a modified post- operative surveillance protocol. Methods: 16 patients were operated for free antero-lateral thigh flap to reconstruct complex soft tissue defects with a close clinical follow up protocol for post operative care depending on the attending personnel in the Plastic surgery unit, Suez Canal University hospital, Ismailia, Egypt. Aesthetic outcome was assessed using a questionnaire based on Posch et al. 2005, including the following items colour, contour, presence of hair, overall appearance and donor site scar. Results: The patients' assessed aesthetic outcome was acceptable in majority of the cases; median score was 4 for all assessed items. Complete flap loss occurred in one case, other complications as arterial thrombosis and hematomas and infection were detected and managed accordingly with flap salvage in the 3 complicated cases. Conclusion: The result suggests that the proposed protocol is sufficient as an alternative. The aesthetic outcome assessed by the patient and the failure rate was in line with other studies.
\end{abstract}

Keywords: Free Flap Surgery, Free Antero-Lateral Thigh Flap, Binocular Single Refraction Magnifying Glasses, Aesthetic Outcome

\section{Introduction}

Reconstruction of complex soft tissue defects and extensive soft tissue defects is a challenging problem. Because selected patients require these procedures, the outcome assessment poses always a problem with discrepancy between how surgeon interprets the results to the patients' opinion which can be totally different. Low incidence of free flap failure [8] nowadays makes the evaluation of the outcome more challenging emphasizing the need for assessing the outcome aesthetically or functionally. Some tools for assessment of aesthetic outcome were developed and are evaluated on several patients groups.[14,15]

The antero-lateral thigh flap was first reported for postburn reconstruction (Song et al., 1984) [3], and since then has become particularly popular among surgeons from China, Taiwan and Japan for reconstruction of complex head and neck defects [4-7]. Over the last 10 years, the free anterolateral thigh flap has been used for a variety of wounds requiring free fascio-cutaneous flap cover in different body regions applying principle of perforator flap harvest.

The economical and financial situation in a developing country like Egypt is not always securing enough funds to meet the increasing demands of the health care field 
forupdating devices and equipment which is considered as a major obstacle for the health care professionals especially in certain types of surgery like micro-vascular surgery which needs advanced equipment for magnification and for intra and post operative monitoring, in a trial to handle these deficiencies we developed (Plastic surgery unit-Suez Canal University hospitals) our own protocol [1] which suits our limited facilities and equipment including a modified tool to assess the outcome of surgery.

As an alternative to the widely used surgical microscope, we applied the use of binocular single-refraction magnifying glasses[1] which was used mainly in relatively large calibre micro-vascular anastomosis to a smaller calibre type of micro-anastomosis as in the case of perforator based flaps as free Antero-lateral thigh flap (ALT).

A training program was implemented according the guidelines published by Mardini et al. [2] including residents' physicians and nurses. It was modified [1] by thorough monitoring of the early post operative period after free ALT flap surgery to substitute for advanced methods of post operative monitoring as laser Doppler imaging.

We aimedto assess the aesthetic outcome of surgical reconstruction by free ALT flap using binocular singlerefraction magnifying glasses and a modified post- operative surveillance protocol.

\section{Subjects \& Methods}

This work was done in Suez Canal University Hospitalas a prospective case series study from July 2011 - May 2012 (16)cases were operated on by the same surgical team. Informed written consents and approval of the local ethical committeewere obtained.The study population is composed of patients with complex soft tissue defects presenting to Plastic Surgery Unit inSuez Canal University Hospital.

\subsection{Inclusion Criteria}

Soft tissue defects (the base of the defect is not liable to be reconstructed with split or full thickness skin graft), age between 12 and 60 years.

\subsection{Exclusion Criteria (Contraindications for the Operation)}

Severe status of chronic illness such as severediabetes mellitus, hypertension, chronic renal failure, severe mental or psychological disorders, andbilateral vascular injuries in the thigh region.In addition, inability to complete the follow up and rehabilitation program as well as smokers were excluded from the study.

\subsection{Surgical Procedure}

16 cases were included in the study with complex soft tissue defects in different body sites, free antero-lateral thigh flap was the procedure in all cases [8] and using binocular single-refraction magnifying glasses, all the operations were done by the same group of surgeons and the same operative theatre. Anastomosis was done using (Proline $7 \backslash 0$ ) interrupted sutures. The optical principals for the binocular singlerefraction magnifying glasses follow the same principles described by Abbas et al. [1]

\subsection{Post-Operative Follow up Protocol}

Part of the subcutaneous tissue of the flap is left exposed for monitoring of the flap viability and delayed coverage after 1 week is done either by skin grafting or primary closure.

\subsubsection{First 24 Hours}

- In the first 2 hours the nurse monitors the perfusion signs of the flap every 5-10 minutes, after 2 hours the monitoring is done twice an hour

Colour: Pink-Pale-bluish

Swelling: minimal-moderate-severe

Temperature: warm-cold-dead cold

Capillary refill: 2-3 seconds- shorter-longer

- Clinical observation of perfusion signs done by the resident physician every hour.

- Every 4 hours: by the resident physician

- Pinprick test: Bright red- dark venous

- Hand held Doppler (8Mhz) is used to detect the flow in the free flap vascular pedicle (Intra-operative marking of the pedicle site is done).

\subsubsection{Second 48 Hours Follow up}

Clinical observation of perfusion signs: (every 4 hours) by the nurse

Every 6 hours:

Pinprick test: Bright red- dark venous

Hand held Doppler ( $8 \mathrm{Mhz}$ ) is used to detect the flow in the free flap vascular pedicle.

\subsubsection{The Next 4Days}

Clinical observation of perfusion signs: (every 6 hours)

Every 12 hours:

Pinprick test: Bright red- dark venous

Hand held Doppler ( $8 \mathrm{Mhz}$ ) is used to detect the flow in the free flap vascular pedicle.

\subsection{Aesthetic Outcome Assessment}

Assessment was done by both the patients individually and panel consisting of 5 independent plastic surgeons, scoring the presence of flap bulkiness, colour mismatch, contour defect, flap contracture and flap sagging. $[13,14]$

- We modified the flap characteristics to be evaluated according to our expert's opinion. Our evaluation sheet included the following flap characteristics:

- Colour match.

- Contour.

- Presence of hair on the flap surface.

- Overall appearance of reconstructed area.

- Donor site scar.

- Both the patient and the panel were asked to score satisfaction with each of the flap characteristics using a 
5 point scale $(1=$ very unsatisfied, $2=$ dissatisfied, $3=$ neither satisfied nor dissatisfied, $4=$ satisfied, $5=$ very satisfied).

- The final aesthetic outcome for each patient was calculated as the mean of scores for all 5 flap characteristics. Final aesthetic outcome $\geq 4$ was considered acceptable. Final aesthetic outcome $<4$ was considered unacceptable.

- Our score was validated by conducting a pilot study over 4 patients.

- The evaluation sheet was translated to Arabic so as to be used by the Egyptian patients. Validation of this translation was done by translation retranslation method.

- Donor site morbidity was assessed regarding motor or sensory deficits, and whether it was closed primarily or not.

\subsection{Data Analysis}

Data was collected from patients in the form of a written questionnaire, and pre and post reconstruction (Photos) Data was processed by STATISTICA 10 by StatSoft Inc. computer package. Wilcoxon Matched Pairs Test was used to test the differences between patients' and surgeons panel aesthetic evaluation. P-values $<0.05$ were considered significant.

\section{Results}

The study comprises 16 cases with complex soft tissue defects with age ranged between 18 and 39 years with a mean age of $27.4 \pm 5.4$ and a male predominance (87\%).(figure 1)

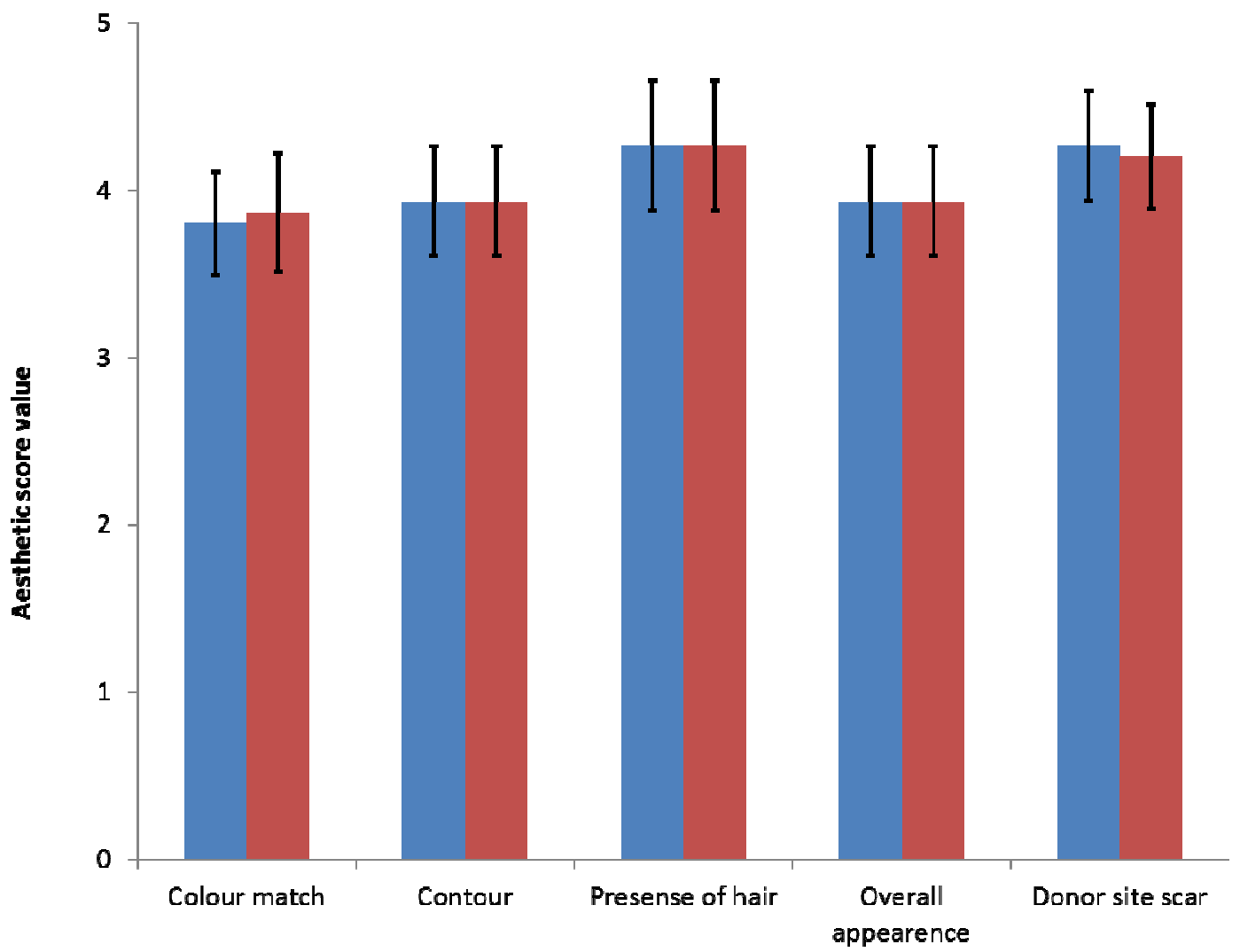

Figure 1. The aesthetic outcome evaluation of the flap done by the patient (blue) and the surgeons' panel (red). Bars $=$ mean, whiskers $=95 \%$ CI. $n=15$, one case omitted because of flap loss.

Table 1. overview of the studied group.

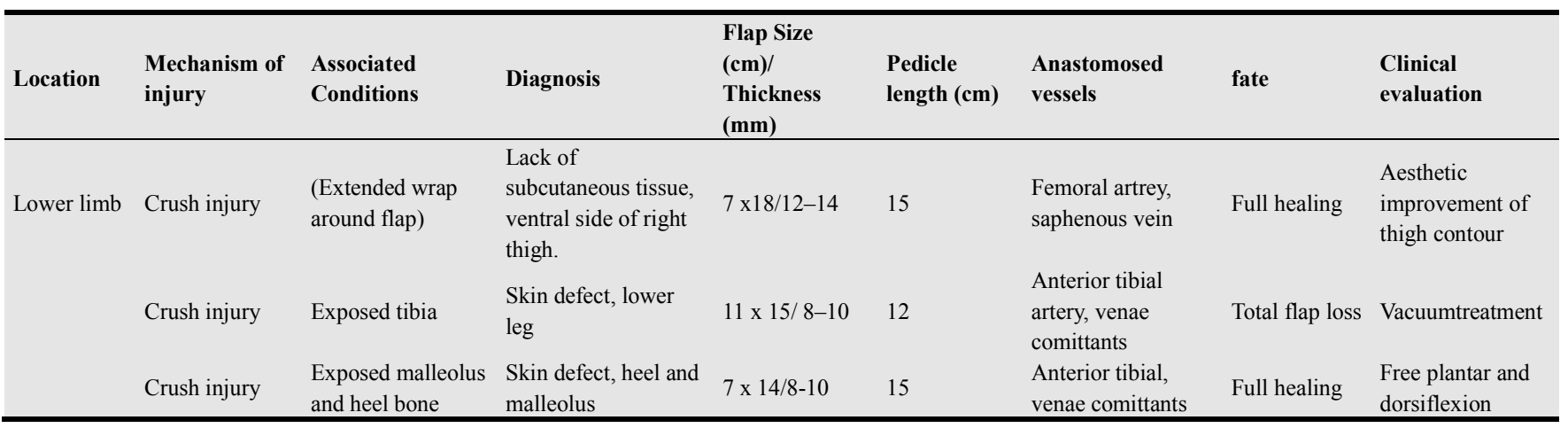




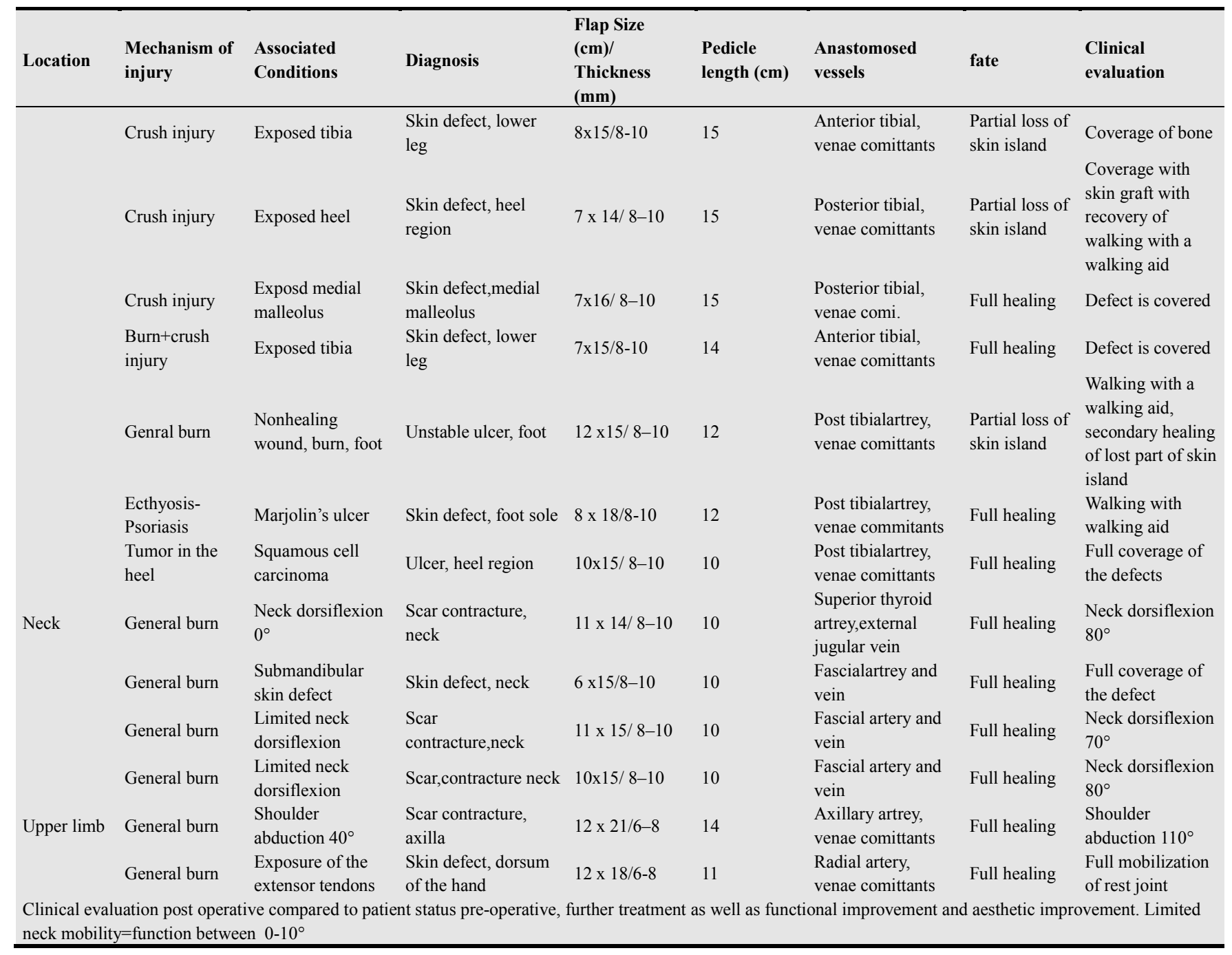

Table 1 data of all the patients included in the study

Table 1 shows the Mechanism of injury, Associated Conditions, Diagnosis, Location, Flap Size (cm), Thickness $(\mathrm{mm})$, Pedicle length $(\mathrm{cm})$, Anastomosed vessels, fate and clinical evaluation.
There was one case of total flap loss among the studied group. Other complications like arterial or venous thrombosis, hematoma or infections are listed in Table 2 as well as the way they were treated.

Table 2. Showing complications\& their management in the study group

\begin{tabular}{llll}
\hline Complications & N & Management \\
\hline Thrombosis & $\begin{array}{l}\text { Arterial } \\
\text { Venous } \\
\text { Donor site } \\
\text { Recipient site } \\
\text { Donor site } \\
\text { Recipient site }\end{array}$ & 2 & $\begin{array}{l}\text { Re-exploration and flap salvage } \\
\text { Total flap loss }\end{array}$ \\
$\begin{array}{l}\text { Infection } \\
\text { Flap loss }\end{array}$ & 1 & Resolution of hematoma conservatively \\
Total & 1 & $\begin{array}{l}\text { Resolution by antibiotic treatment according to culture and sensitivity } \\
\text { Vacuum treatment followed by skin grafting }\end{array}$ \\
\hline
\end{tabular}

$\mathrm{N}=$ number of complications which occurred in 4 patients.

Study group complications

The donor sites in all cases (16 cases) of the study group were closed primarily with no need of skin grafts.

\subsection{Aesthetic Outcome Evaluation}

The results of the aesthetic outcome evaluation of the flap done by the patient and the surgeons' panel are presented in figure I. Four of the 15 patients assessed themselves with unacceptable results regarding colour match, 3 were dissatisfied regarding contour and overall appearance, while 1 patient was dissatisfied with his donor scar.Both patients and surgeons scored 4 on the median level in all items and 
there were no differences between patients and surgeons evaluation ( $p$-values $\geq 0.68$ ).

\subsection{Case Report}

36 years old female who suffered a third degree burn 6 years ago and had developed a severe neck contracture

hindering the movement of the neck in all directions with 0 degrees dorsi-flexion. Multiple attempts for release of this contracture with split thickness skin grafts were done with non-satisfactory results and decision for release and use of a free anterolateral thigh flap was taken.

\subsection{Pre-Operative Photos}

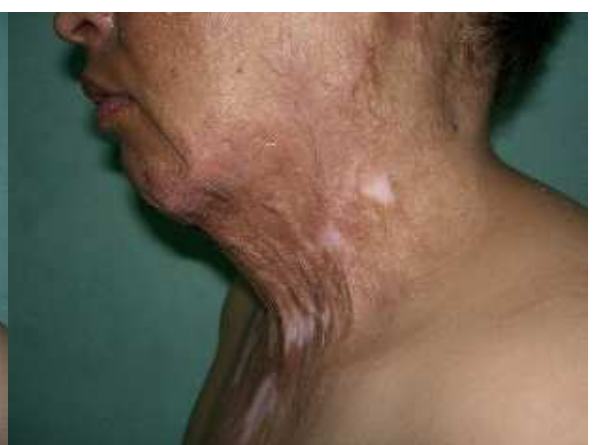

Figure 2. Front view\& side view of post burn contracture.

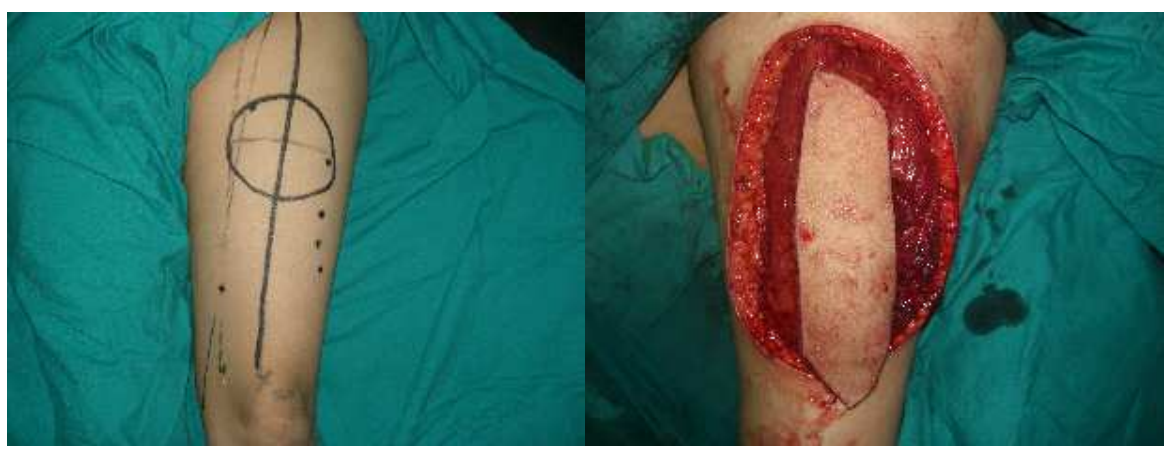

Figure 3. Skin markings for perforators of anterolateral thigh flap located by hand Doppler and flap dissection.

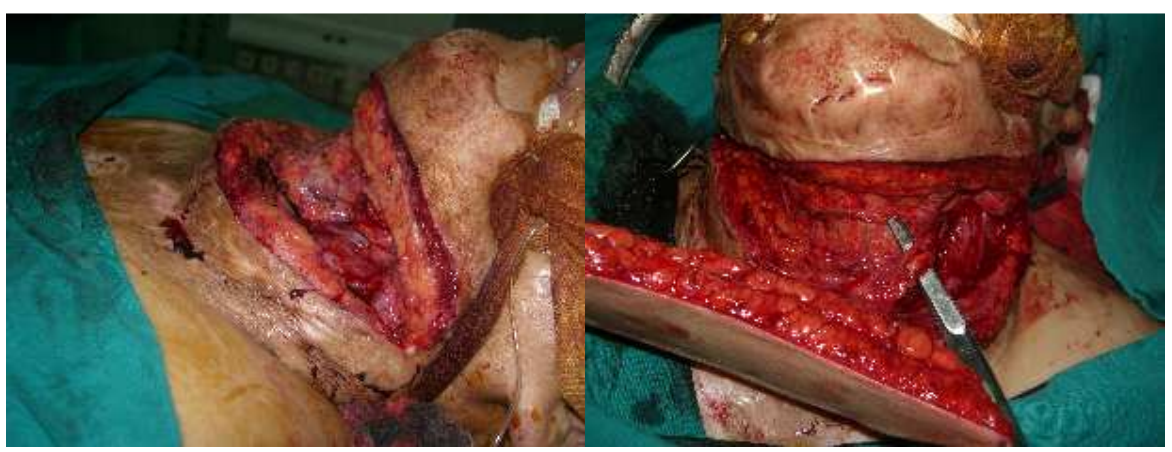

Figure 4. The neck after full release (Lateral view) and anastomosis of the flap pedicle to the superior thyroid artery and veins.

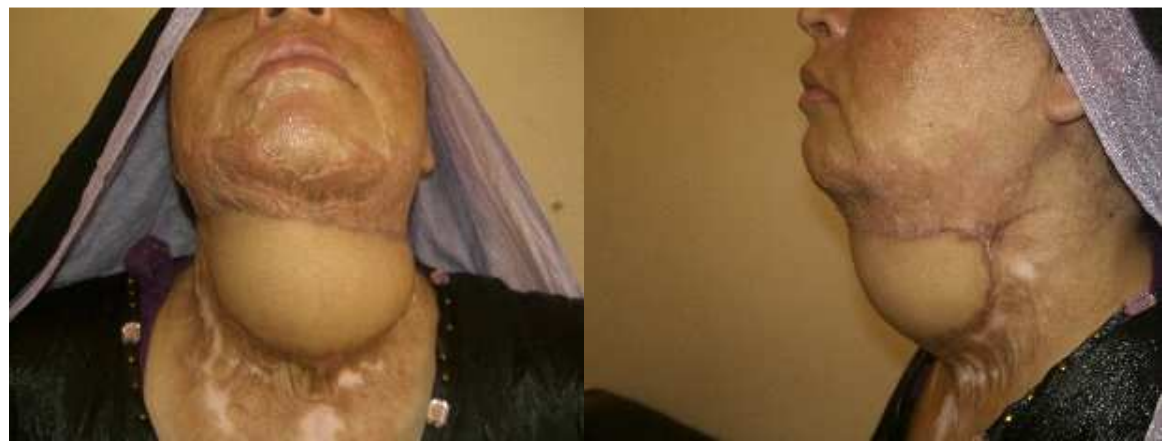

Figure 5. Post- operative 3 weeks (anterior \& lateral view). 


\section{Discussion}

The result of the study suggests that the proposed protocol has shown to be sufficient as an alternative based on the outcome of free ALT flap with only one failure and acceptable aesthetic outcome based on a patient and surgeons' panel assessment. The result is further strengthened by the similarity between the surgeons' panel assessment and the assessment done by the patient regarding the aesthetic outcome.

The failure rate in the study was similar to previous studies $[8,9]$, the rate of complication was lower compared to other studies [10,12]. The early detection of post- operative complications allowed dealing with them in a proper manner and leads to flap salvage in 3 cases which is a good effect of the post-operative protocol.

The aesthetic outcome results by the patients and the surgeon panel wascongruent, indicating the clearance of the outcome and the good preparation of the patient knowledge pre-operatively that the patients are aware of the exact expectations. The proportion of the patients who were content with the aesthetic results of the operation was comparable to the results previously published studies using the same tool. [13]

The absence of a control group is a limitation for the study as well as the few numbers of cases. Strength of the study is that it was done in one centre with a tight control of the protocol including the surgical team and technique and outcome assessment. The functional outcome was not systematically assessed however clinical notes of improvement of function were presented to substitute for this.

\section{Conclusion}

The result suggests that the proposed protocol is sufficient as an alternative. The aesthetic outcome assessed by the patient and the failure rate was in line with other studies.

\section{References}

[1] Ashraf H. Abbas, Waleed A. Ghobashy ,Amr M. Moghazy Binocular single-refraction magnifying glasses for free flap surgery: a reliable method for developing countries. Eur J PlastSurg (2012) 35:521-525.

[2] Christopher J. Salgado, Harvey Chim, ShaylaSchoenoff, P.A.C. and Samir Mardini,Postoperative care and monitoring of the reconstructed head and neck patient. Seminars in Plastic surgery (2010) / Vol. 24, Number. 3:281-287.

[3] Song YG, Chen GZ, Song YL. The free thigh flap: A new free flap concept based on the septocutaneous artery. $\mathrm{Br} J$ PlastSurg. 1984;37:149-159.
[4] Demirkan F, Chen HC, Wei FC et al. (2000). The versatile anterolateral thigh flap: a musculocutaneousflap in disguise in head and neck reconstruction. British Journal of Plastic Surgery, 53: 30-36.

[5] Kimata Y, Uchiyama K, Ebihara S et al. (1997). Versatility of the free anterolateral thigh flap for reconstruction of head and neck defects. Archives of Otolaryngology, Head and Neck Surgery, 123:1325-1331.

[6] Koshima I, Fukuda H, Yamamoto H, Moriguchi T, Soeda S, Ohta S (1993a). Free anterolateral thigh flaps for reconstruction of head and neck defects. Plastic and Reconstructive Surgery, 92: 421-430,\ Koshima I, Yamamoto H, Hosoda M, Moriguchi T, Orita Y, Nagayama H (1993b). Free combined composite flaps using the lateral circumflex femoral system for repair of massive defects of the head and neck regions: an introduction to the chimeric flap principle. Plastic and Reconstructive Surgery, 92: 411-420.

[7] Luo S, Raffoul W, Luo J, Gao J, Chen L, Egloff DV (1999). Anterolateral thigh flap; a review of 168 cases. Microsurgery, 19: $232-238$

[8] Fu-Chan Wei, Vivekjain, NaciCelik, Hung-chi chen, David chewi-chin chuang and chih-hunglin.(2002), Have we found an ideal soft-tissue flap? An experience with 672 Anterolateral thigh flaps, PlastReconstr Surg. June 2002;2219-2226.

[9] Abd El-hamid Abdel-khalek; Abdul-Mohsen Allam; Amgad Hendy; Sayed Abdel-Razek; HashemAyad; SobhyHweidy; HelmyShalaby and MoustafaHegazy, Clinical Evaluation of Free Anterolateral Thigh Flap in the Reconstruction of Major Soft Tissue Defects in the Leg and Foot. Egypt. J. Plast. Reconstr. Surg., Vol. 27, No. 2, July: 173-180, 2003.

[10] Wei, F. C., Demirkan, F., Chen, H. C., et al. Double free flapsin reconstruction of extensive composite mandibular defectsin head and neck cancer. Plast. Reconstr. Surg. 103: 39, 1999.

[11] Gabr, E., Kobayashi, M. R., Salibian, A. H., et al. Mandibularreconstruction: Are two flaps better than one? Ann. Plast.Surg. 52: 31, 2004.

[12] Jeng, S. F., Kuo, Y. R., Wei, F. C., et al. Reconstruction of extensive composite mandibular defects with large lip involvementby using double free flaps and fascia lata grafts for oral sphincters. Plast. Reconstr. Surg. 115: 1830, 2005.

[13] Mureau MA, Posch NA, Meeuwis CA, Hofer SO. Anterolateral thigh flap reconstruction of large external facial skin defects: a follow-up study on functional and aesthetic recipient- and donor-site outcome. Plastic and reconstructive surgery 2005; 115: 1077-1086.

[14] Posch NA, Mureau MA, Flood SJ, Hofer SO. The combined free partial vastuslateralis with anterolateral thigh perforator flap reconstruction of extensive composite defects. British journal of plastic surgery 2005; 58: 1095-1103.

[15] Posch NA, Mureau MA, Dumans AG, Hofer SO. Functional and aesthetic outcome and survival after double free flap reconstruction in advanced head and neck cancer patients. Plastic and reconstructive surgery 2007; 120: 124-129. 